\title{
TRAITE A LA MACHINE DES BREBIS PRÉALPES DU SUD. IMPORTANCE DES DIFFÉRENTES OPERATIONS DE IAA TRAITE
}

\author{
G. RICORDEAL, J. MARTINET, R. DENAMUR \\ Aver la collaboration technique de P. P'etregcts et de M. Carpexter \\ Station centrale de Génétique animale; Laboratoire de Physiologie de la Laciation, \\ Centre national de Recherches zoolechniques, Jouy-en-Josas (Seine-el-Oise)
}

\section{SOMMAIRF,}

Les brebis Préalpes $d u$ Sud utilisées dans ces expériences ont été traites à la machine 2 fois par jour. Chaque traite comprenait 3 opérations :

$\left.\mathbf{I}^{\mathrm{D}}\right)$ la " traite machine " proprement dite, de la pose des gobelets à la fin de l'écoulement du lait ;

$2^{o}$ ) un égouttage machine avant l'enlèvement des gobelets ;

$\left.3^{\circ}\right)$ un égouttage manuel ou "repasse".

L'importance de la quantité de lait obtenue au cours de ces 3 opérations, exprimée en p. Ioo du total de lait trait, varie du $64^{\mathrm{e}}$ au $164^{\mathrm{e}}$ jour de lactation de $: 68$ a 59 p. Ioo pour la traite machine, 23 à $28 \mathrm{p}$. 100 pour l'égouttage machine; 9 à I $3 \mathrm{p}$. Ioo pour l'égouttage manuel. Malgré ces opérations successives, la mamelle n'est pas complètement vidée : le volume de lait résiduel que l'on peut obtenir après injection d'une $U$. I. d'ocytocine représente encore I 3 à $23 \mathrm{p}$. Ioo de la quantité de lait traite antérieurement ( 32 brebis en expérience).

La durée moyenne de la " traite machine " est de I mn $26 \mathrm{~s}$, celle de l'égouttage machine de $32 \mathrm{~s}$.

L'enregistrement hebdomadaire de la descente du lait de 32 brebis, a permis de distinguer 2. phases : écoulement du lait citernal au cours des I 5 premières secondes, puis phase d'évacuation sous l'action de l'ocytocine libérée par le réflexe neuro-endocrinien d'éjection du lait (30 à $45 \mathrm{~s}$ ). L'importance du phénomène d'éjection diminue avec le stade de lactation.

L'étude de l'égouttage manuel a été effectuée avec les données de $3 \mathbf{I} 3$ lactations. Le volume total de lait obtenu à l'égouttage manuel sur l'ensemble de la période de traite $(9,3$ litres de lait en moyenne, soit I I,2 p. Ioo du volume total de lait obtenu à la machine) est plus élevé en première année de traite, mais varie peu au cours des lactations suivantes; il ne dépend pas de façon significative de la durée d'allaitement, du mode de sevrage ou du nombre d'agneaux allaités ; son coeffcient de variabilité journalière est de $76 \mathrm{p}$. Ioo; son coefficient de répétabilité d'une lactation it l'autre est de 0,28 sans correction des données et de o,53 après correction.

Le volume iournalier de l'égouttage manuel décroît rapidement au cours des 2 premières semaines de traite, puis reste pratiquement constant par la suite. Ta quantité de matière grasse obtenue à l'égouttage manuel représente (en fonction du stade de lactation) 24 à $30 \mathrm{p}$. 100 de celle du lait trait ì la machine.

$\mathrm{La}$ suppression de l'égouttage manuel aboutit en moyenne à une diminution de $29 \mathrm{p}$. Ioo du potentiel sécrétoire pour les brebis ayant allaité leurs agneaux i ou 4 semaines. Par contre, la pratique d'un égouttage manuel supplémentaire à chaque traite permet d'obtenir un gain de production de 15 p. Ioo.

Les relations entre la production totale de lait obtenue à la traite et les principales caractéristiques de la traite ont été étudiées. 


\section{INTRODUC'TION}

L'objet de cette étude est d'observer le rythme suivant lequel les brebis donnent leur lait au cours de la traite. Ce rythme est susceptible de fournir des indications sur le phénomène d'éjection, l'importance et l'efficacité de chacune des opérations de la traite.

I1 existe peu de travaux sur cette question. En Israël, GrUNFEI, VolcanI et EYAL (I958) ont observé les quantités de lait obtenues successivement au cours de la traite machine, l'égouttage machine et l'égouttage manuel. Sur I $_{5}$ brebis Awasi donnant de 6 à $900 \mathrm{ml}$ de lait par traite, ces auteurs enregistrent environ $60 \mathrm{p}$. Ioo de la production au cours de la première opération, i7 p. Ioo au cours de la seconde et 23 p. Ioo au cours de la troisième.

En Italie, sur 3 I brebis (ientile de P'uglia contrôlées régulièrement au cours de la lactation, SAI ERNo (I935) enregistre à l'égouttage manuel I7 à 44 p. Ioo de la production obtenue lors de la traite manuelle qui le précède.

En France, la technique traditionnelle de traite peut certainement être modifiée, en vue d'obtenir à la fois, une économie de main-d'œuvre et un rendement maximum des mécanismes physiologiques mis en jeu au cours de la traite. Ces objectifs ne peuvent être atteints que si l'on connait bien ces mécanismes chez la brebis.

\section{MATÉRIEL F'T MÉTHODES}

\section{Matíriel}

Les brebis laitières de race "Préalpes du Sud" utilisées pour nos expériences (à la ferme de Brouessy) ont été réparties dans des lots qui se distinguent par les conditions de sevrage, de traite et par le nombre d'agneaux allaités.

\section{Technique de traite}

La traite a lieu deux fois par jour. Les gobelets de la machine sont posés sans massage préalable de la mamelle. Lorsque cesse l'écoulement du lait dans le récipient collecteur, le trayeur pratique un " égouttage machine" en massant énergiquement la glande, les gobelets étant encore en place. Linfin, dans le but de réaliser un assèchement aussi complet que possible de la mamelle, un trayeur spécialisé effectue ensuite un égouttage manuel ou "repasse".

\section{Cinétique de la descente du lait au cours de la traite machine}

Un observateur enregistre toutes les ${ }_{5} 5$ secondes la cinétique du remplissage d'un réservoir gradué, placé près de la glande mammaire afin de réduire le volume mort constitué par les canalisations.

Les contrôles sont effectués une fois par semaine, à la traite du matin. L'ordre des opérations est le suivant :

La pose des gobelets donne le temps zéro $\left(t_{0}\right)$; 
L'opérateur attend le remplissage des canalisations, l'arrivée du premier jet de lait dans le réservoir gradué donne le temps $t_{1}$; ment.

A partir de $t_{1}$ l'opérateur mesure le niveau du lait toutes les ${ }_{5} \mathrm{~s}$ jusqu'à ce que cesse l'écoule-

\section{Lait résiduel}

Le lait résiduel est mesuré une fois par semaine, à la traite du soir, après injection intraveineuse de I U. I. d'ocytocine (Syntocinon).

\section{Caractéristiques de la machine à traire}

Marque Alfa-Laval. Vide de $33 \mathrm{~cm}$ de mercure. Vitesse de pulsation : $180 / \mathrm{mm}$. Rapport $\mathrm{I} / \mathrm{r}$.

Gobelets pesant environ $240 \mathrm{~g}$, sans leur canalisation. Corps du gobelet : $\mathrm{I} 44 \mathrm{~g}$, diamètre $30 \mathrm{~mm}$, hauteur r $10 \mathrm{~mm}$; pipe inférieıtre : $63 \mathrm{~g}$; caoutchouc : $33 \mathrm{~g}$, diamètre entrée : $20 \mathrm{~mm}$, diamètre du corps : $20 \mathrm{~mm}$.

\section{RÉSULTATS}

L'étude de la traite machine, de l'égouttage machine et du lait résiduel porte uniquement sur 32 brebis. Les contrôles sont hebdomadaires de la $9^{e}$ à la $23^{e}$ semaine de lactation. Les caractéristiques moyennes de la traite de ces brebis sont résumées dans les tableaux I et 2.

\section{TABLEAU I}

Caractéristiques moyennes de la traite de 32 brebis Préalpes controlées $d u 64^{\mathrm{e}}$ au $164^{\mathrm{e}}$ jour de lactation

\begin{tabular}{|c|c|c|}
\hline Variables & Moyenne & Limites de variation \\
\hline Lait total ........... & 701 & $43-112$ \\
\hline $\begin{array}{l}\text { Duree totale de lactation } . . . \ldots \ldots \ldots \ldots \ldots \ldots \ldots \ldots \\
\text { Persistance }\end{array}$ & $193 i$ & $161-20$ \\
\hline 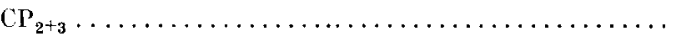 & 0,950 & $0,90 \div-0.987$ \\
\hline 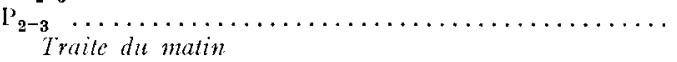 & $295 \mathrm{ml}$ & $107-538$ \\
\hline Lait machine $\ldots \ldots \ldots \ldots \ldots \ldots \ldots \ldots \ldots \ldots \ldots$ & $217 \mathrm{ml}$ & \\
\hline Iait de l'égouttare machine ................... & $80 \mathrm{ml}$ & $43-139$ \\
\hline 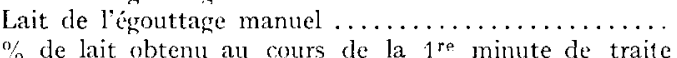 & $36 \mathrm{ml}$ & $17-69$ \\
\hline 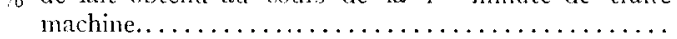 & $82 \%$ & $71-95$ \\
\hline $\begin{array}{l}\text { Débit ma.hine/minute } \ldots \ldots \ldots \ldots \ldots \ldots \ldots \ldots \ldots \ldots \\
\text { Iraile du soir }\end{array}$ & $15 \mathrm{~L} \mathrm{ml} / \mathrm{mm}$ & \\
\hline lait résiduel. $\ldots \ldots \ldots \ldots \ldots \ldots \ldots \ldots \ldots \ldots \ldots$ & $61 \mathrm{l11}$ & $21-150$ \\
\hline
\end{tabular}

$\left(\mathrm{P}_{\mathrm{Z}+3}=\right.$ moyenme des coefficients de persistance hebdomadaires pour les périodes 2 et 3 . Le CP hebdomadaire est le rapport entre la production de la semaine d'ordre $(n+1)$ à celle de la semaine d'ordre $(n)$.

$\mathrm{P}_{2-3}=$ différence moyenne de production entre la ${ }^{-\mathrm{e}}\left(50^{\mathrm{e}}\right.$ au $100^{\mathrm{e}}$ jour de lactation) et la $3^{\mathrm{e}}$ $\left(100^{\mathrm{e}} \text { au } 150\right)^{\mathrm{e}}$ jour) période de lactation. 
TABLEAU 2

Importance des différentes opirations de la traite en fonction du stade de lactation ( $3^{2}$ brebis)

\begin{tabular}{|c|c|c|c|c|c|c|c|c|c|c|c|c|c|}
\hline \multirow{3}{*}{$\begin{array}{c}\text { Semaines } \\
\text { de } \\
\text { lactation }\end{array}$} & \multirow{3}{*}{$\begin{array}{c}t_{1}-t_{0} \\
S\end{array}$} & \multicolumn{4}{|c|}{ Traite machine } & \multicolumn{3}{|c|}{$\begin{array}{l}\text { Égouttage } \\
\text { machine }\end{array}$} & \multirow{2}{*}{\multicolumn{2}{|c|}{$\frac{\begin{array}{c}\text { Egouttage } \\
\text { manuel }\end{array}}{\text { Lait }}$}} & \multirow{3}{*}{$\begin{array}{c}\text { Total de } \\
\text { la traite } \\
\text { matin } \\
\quad \text { ml }\end{array}$} & \multirow{2}{*}{\multicolumn{2}{|c|}{$\begin{array}{l}\text { Iait rési- } \\
\text { duel apres } \\
\text { traite du } \\
\text { soir }\end{array}$}} \\
\hline & & \multicolumn{2}{|c|}{ Lait } & \multirow{2}{*}{$\begin{array}{c}\text { Durée } \\
s\end{array}$} & \multirow{2}{*}{$\begin{array}{c}\text { Débit } \\
\mathrm{ml} / \mathrm{mnn}\end{array}$} & \multicolumn{2}{|c|}{ Lait } & \multirow{2}{*}{ Durée } & & & & & \\
\hline & & $\mathrm{ml}$ & $\%$ & & & $\mathrm{ml}$ & $\%$ & & $\mathrm{ml}$ & $\%$ & & $\mathrm{ml}$ & $\%$ \\
\hline 9 & 7 & $32 z$ & 68 & 91 & 214 & 112 & 23 & 26 & 46 & 9 & 483 & 63 & 13 \\
\hline 10 & 8 & 317 & 68 & 98 & 196 & 98 & 21 & 39 & 43 & 9 & 468 & 61 & 13 \\
\hline 11 & 15 & 306 & 68 & 88 & 209 & 98 & $2 y$ & 36 & $4 t^{\prime}$ & 10 & 148 & $6 x^{\prime}$ & $1 ' t$ \\
\hline 12 & 7 & 28.3 & 67 & 101 & 168 & 91 & 22 & 35 & 47 & 11 & 421 & 72 & 17 \\
\hline 13 & 8 & 284 & 70 & $9 y^{\prime}$ & 181 & 79 & 20 & 33 & 39 & 10 & 402 & 64 & 16 \\
\hline 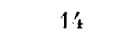 & 8 & 239 & 66 & 95 & 151 & 81 & 22 & 32 & $\$ 1$ & 11 & 361 & 72 & 20 \\
\hline 15 & 5 & 220 & 66 & 88 & 150 & 79 & 23 & 29 & 37 & 11 & 336 & 62 & 18 \\
\hline 16 & 6 & 198 & 63 & 88 & 135 & 8.3 & 96 & 35 & 36 & 11 & 317 & 67 & 21 \\
\hline 17 & 7 & 191 & 63 & 80 & 143 & 78 & 26 & 33 & 32 & 11 & 301 & $6{ }^{\prime}$ & 21 \\
\hline 18 & 8 & 176 & 60 & 82 & 129 & 77 & 26 & 34 & 38 & 13 & 291 & 69 & 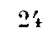 \\
\hline 19 & 9 & 156 & 60 & 79 & 118 & 72 & 28 & 32 & 30 & 12 & 258 & 60 & 23 \\
\hline 20 & 7 & 148 & 61 & 83 & 107 & 65 & 27 & 33 & 29 & 12 & 242 & 57 & 23 \\
\hline 21 & 7 & 153 & 61 & 77 & 119 & 70 & 28 & 33 & 27 & 11 & 250 & $5{ }^{\prime}$ & $\because 2$ \\
\hline 2.2 & 8 & $1: 1$ & 61 & 71 & 119 & 6.1 & 27 & 29 & 28 & 12 & 230 & 48 & 21 \\
\hline 23 & 7 & 123 & 59 & 72 & 151 & 58 & 28 & 29 & 26 & 13 & 207 & $\ell_{4} 1$ & 20 \\
\hline
\end{tabular}

$\left(t_{1}-t_{0}\right):$ durée de remplissage des tuyaux en $s$.

$\%$ : en $\mathrm{p}$. 100 du volume total de la traite du matin.

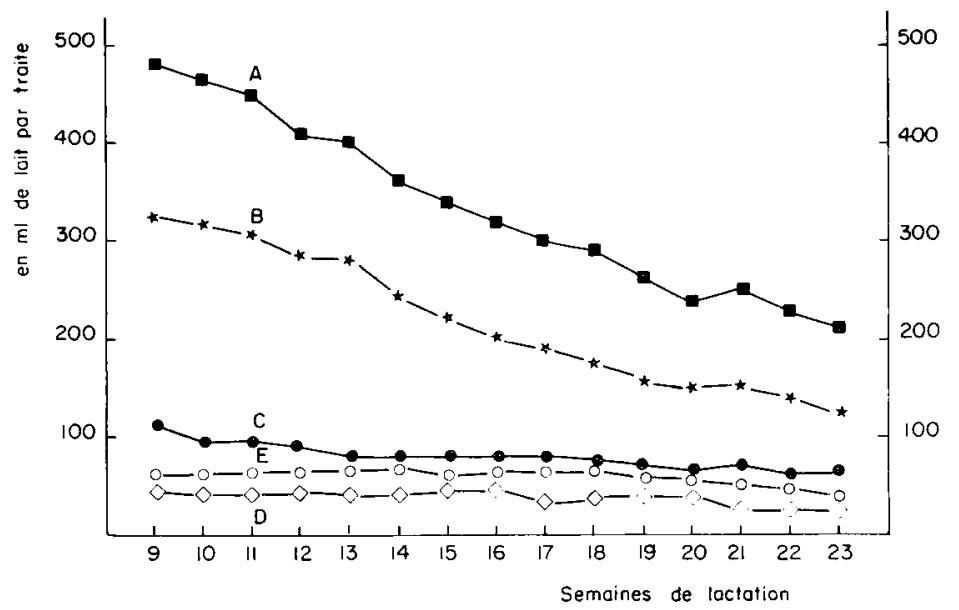

Graph. I. - Importance des différentes phases de la traile complite.

$$
\begin{aligned}
& \text { A - traite totale } \\
& \text { B - traite machine } \\
& \text { C - égouttage machine } \\
& \text { D - égouttage manuel } \\
& \text { E - lait résiduel }
\end{aligned}
$$




\section{A. - I'raite Machine}

I. Temps de remplissage des tuyaux

Il est en moyenne de 8 secondes et varie peu, aussi bien entre brebis qu'entre stades de lactation (tableau 2).

\section{Durée}

La durée moyenne de la traite à la machine entre la $9^{\mathrm{e}}$ et la $23^{\mathrm{e}}$ semaine de lactation est de I mn $26 \mathrm{~s}$. Fille passe de I mn 40 au début de la période de traite à I mn Io en fin de lactation (graphique 2). A chaque stade, l'écart type des durées est de l'ordre de $30 \mathrm{~s}$.
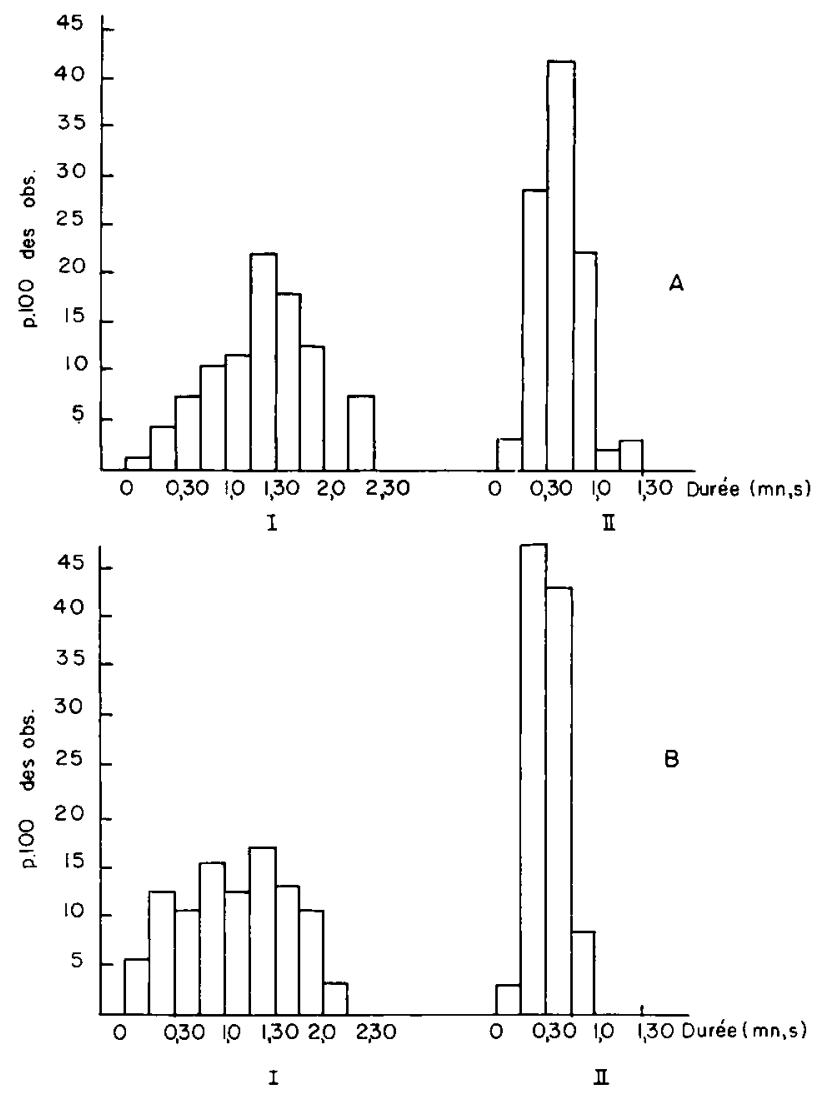

GRAPH. 2. - Distribution des durées de traile

I … Traite machine

II -- Érouttage machine

$A-60$ a 80 jours de lactation

i) -. I 40 à 160 jours de lactation 
La traite machine en elle-même est donc très courte. Le pourcentage de brebis dont l'écoulement du lait se prolonge au-delà de $2 \mathrm{mn}$ est de $\mathrm{I}_{4} \mathrm{p}$. Ioo au $2^{\mathrm{e}}$ mois et de $3 \mathrm{p}$. Ioo seulement au $5^{\mathrm{e}}$ mois. Ce temps de "traite machine " représente 70 à So p. roo du temps de séjour utile des brebis dans la stalle de traite.

\section{Quantité de lait recueillie au cours de la traite machine}

L,e volume de lait obtenu à la traite machine décroît régulièrement de $325 \mathrm{ml}$ à $123 \mathrm{ml}$ avec le stade de lactation. Ces quantités représentent respectivement 67 et 59 p. Ioo du total de la traite (tableau 2). Il est important de souligner que ce volume constitue au cours de la lactation, la fraction la plus variable de la traite.

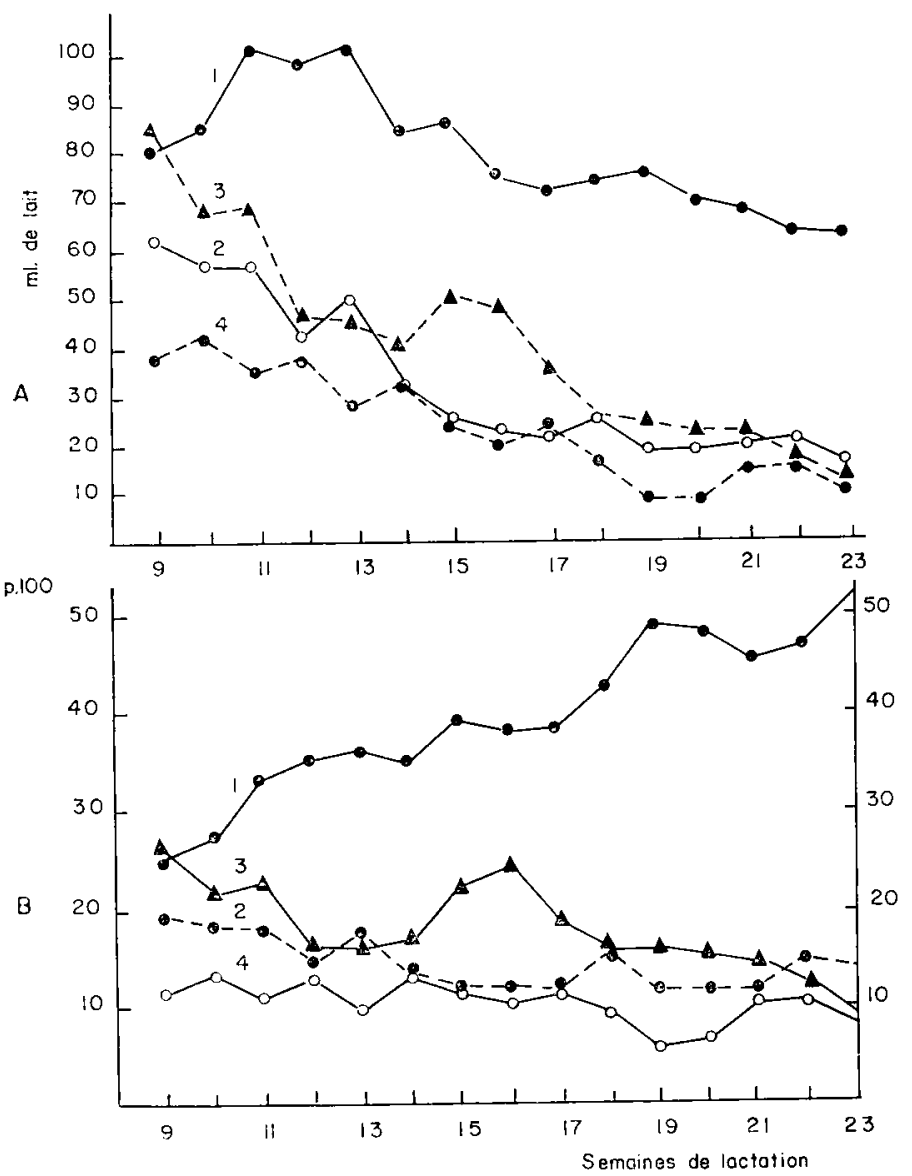

Cirarir. 3. - Ecoulement tu lat au cours de la premibe minute de traite machine.

Fariations ance le slade de lactitisn

A. - en ml de lait

$B$ - en b. roo du volume de la traite machine

I - de o a I 5 s

2 - de I 5 is $30 \mathrm{~s}$

3 - de 30 il 45 s

4 -.. de 45 it 60 s 


\section{Dynamique de la traite machine}

a) Débit moyen.

Calculé sur l'ensemble de la lactation, le débit moyen est de I5I ml par minute à la traite du matin. De $200 \mathrm{ml} / \mathrm{mn}$ au $2^{\mathrm{e}}$ mois, il diminue jusqu'à Ioo $\mathrm{ml} \mathrm{mn}$ vers le $6^{\mathrm{e}}$ mois, traduisant la décroissance de la quantité de lait obtenue à la traite machine (tableau 2).

\section{b) Comparaison des différentes fractions de la traite machine.}

La première fraction ( $0-15 \mathrm{~s})$ reste la plus importante au cours de la lactation. Elle croît de la $g^{\mathrm{e}}$ à la $\mathrm{I} 3^{\mathrm{e}}$ semaine pour décroître ensuite (il serait intéressant de savoir si une telle évolution s'observe sur des brebis traites à la machine dès la mise bas ou si on doit l'attribuer au passage de l'allaitement à la traite machine). Cette première fraction constitue 25 p. roo du lait trait à la machine en début de période de traite et $50 \mathrm{p}$. Ioo en fin de lactation. Elle semble représenter essentiellement le lait citernal que la machine peut extraire dès la pose des gobelets, le réflexe d'éjection n'ayant pas encore eu le temps de se produire.

La deuxième fraction (15-30 s) passe de $60 \mathrm{ml}$ au début de la saison de traite à $20 \mathrm{ml}$ en fin de lactation. Elle n'est intéressante que si nous la comparons à la troisième qui lui est presque toujours supérieure. Cette supériorité, montre qu'un afflux de lait se produit dans la citerne entre la $3^{\mathrm{e}}$ et la $45^{\mathrm{e}} \mathrm{s}$, alors que la deuxième fraction correspondrait à la fin de l'extraction du lait citernal commencée dès la pose des gobelets.

La troisième fraction $(30-45 \mathrm{~s})$ représenterait donc, tant par sa place dans le temps, que par la quantité qui la caractérise, le lait chassé des acini par le réflexe neuro-endocrinien d'éjection. Ce réflexe serait déclenché par la pose des gobelets.

Les quatrième, cinquième et sixième fractions correspondant à la fin de l'extraction du lait à la machine sont en général très réduites.

\section{c) Place du débit maximum.}

Pour estimer les variations individuelles, nous avons essayé de situer, à chaque traite, la place du débit maximum (tableau 3). On constate que plus de 50 p. roo des brebis présentent leur débit maximum au cours des 15 premières secondes de traite. Par ailleurs, le pourcentage d'animaux présentant un deuxième pic est respectivement de 78 et 65 , à la $\mathrm{II}^{\mathrm{e}}$ et à $1 \mathrm{a} \mathrm{I}^{\mathrm{e}}$ semaine. Cette augmentation du débit que l'on peut attribuer au réflexe d'éjection est donc observable sur la majorité des brebis.

\section{B - ÉgoutTage Machine}

La durée de "l'égouttage machine " peut être considérée comme constante au cours de la lactation ( 32 s en moyenne). La quantité de lait qu'il permet d'obtenir décroît cependant de Ioo à $60 \mathrm{ml}$ avec le déroulement de la lactation ( 23 à 28 p. IOO du volume total de la traite, tableau 2). 
mi. de lait
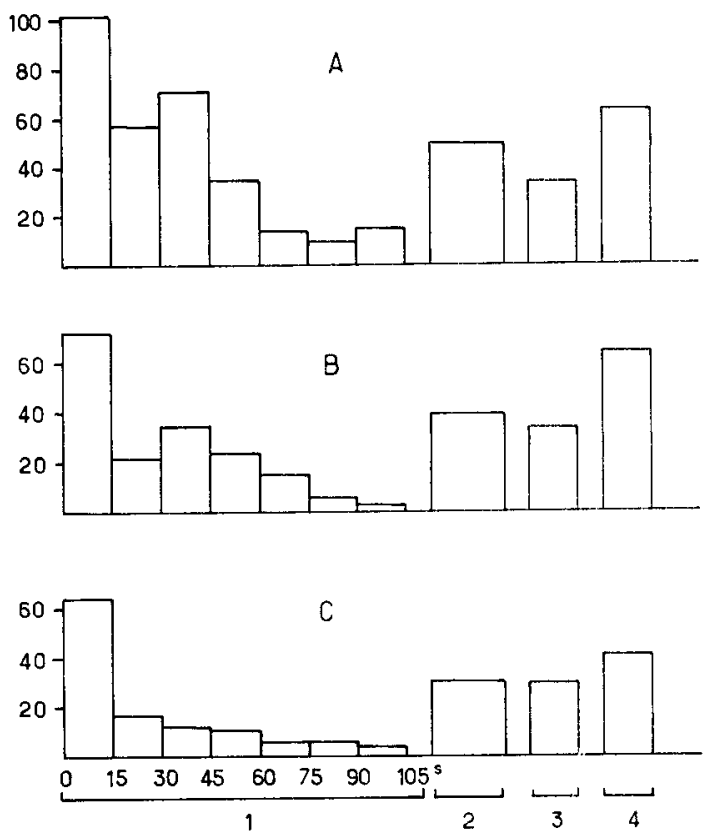

Graph. 4. - Cinétique de la descente du lait au cours de la traite, à 3 stades de la lactation.

$$
\begin{aligned}
& \text { A - I I e semaine de lactation } \\
& \mathrm{B}-\mathrm{I} \mathrm{y}^{\mathrm{e}} \text { semaine de lactation } \\
& \text { I -. Traite machine } \\
& C-23^{\text {e }} \text { semaine de lactation } \\
& \text { I - Egouttage machine } \\
& 3 \text { - Egouttage manuel } \\
& 4 \text { - Lait résiduel }
\end{aligned}
$$

\begin{tabular}{|c|c|c|c|c|c|c|c|c|c|c|c|c|c|c|c|}
\hline \multirow{2}{*}{$\begin{array}{l}\text { Fractions successives } \\
\text { de la traite marhine }\end{array}$} & \multicolumn{15}{|c|}{ Semaines de lactation } \\
\hline & 9 & 10 & 11 & 12 & 13 & $1 / 4$ & 15 & 16 & 17 & 18 & 19 & 20 & 21 & 22 & 23 \\
\hline de 0 à $15 \mathrm{~s}$ & 56 & 50 & 69 & 72 & 72 & 78 & 75 & 78 & 75 & 75 & 97 & 85 & 75 & 78 & 72 \\
\hline de 15 à $30 \mathrm{~s}$ & 12 & 12 & 6 & 6 & 6 & $\ldots$ & 3 & 3 & 12 & 12 & 3 & 6 & 12 & 6 & 9 \\
\hline de 30 à $45 \mathrm{~s}$ & 22 & 10 & 15 & 15 & 10 & 10 & 6 & 12 & 10 & 12 & - & 6 & 6 & 10 & 13 \\
\hline de 45 à $60 \mathrm{~s}$ & 3 & 10 & 6 & - & 一 & 一 & 10 & 一 & 一 & - & - & 3 & 6 & 6 & 6 \\
\hline
\end{tabular}

\section{TABLEAU 3}

Pourcentage de brebis présentant leur débit maximum dans les différentes fractions de la $\mathbf{I}^{\mathrm{re}}$ minute de traite 


\section{C - Égouttage manuel}

L'égouttage manuel est couramment pratiqué dans tous les élevages de la zone de Roquefort. Cette opération augmente la durée totale de traite et exige un personnel supplémentaire qualifié. Il est donc important de connaître, les quantités de lait qu'il permet d'obtenir ainsi que son action à long terme sur la production totale et la persistance de la lactation.

Nos observations sur l'égouttage manuel portent sur 3 I3 lactations dont la répartition est indiquée dans le tableau 4.

\section{I. - Quantités de lait obtenues à l'égouttage manuel par lactation}

La quantité moyenne de lait obtenue à l'égouttage manuel pendant une lactation est de 9,3 litres; elle varie de 2 à 26 litres (graphique 5 A). La distribution de ces quantités est légèrement dissymétrique :
20 p. Ioo des brebis ont moins de 6 litres;
62 p. Ioo donnent entre 6 et I2 litres;
I5 p. Ioo donnent entre I2 et I 8 litres;
3 p. Ioo ont plus de I8 litres de lait.

L'importance relative de la quantité de lait obtenue à l'égouttage manuel par rapport à celle obtenue à la machine est exprimée par le pourcentage de " repasse " (P. R.). Ce pourcentage a une distribution également dissymétrique de moyenne $I I, 2$ (graphique $5 \mathrm{~B}$ ).

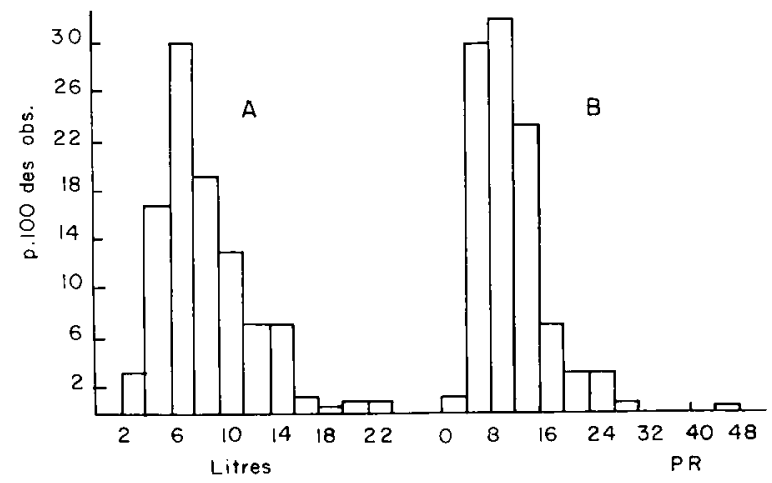

GRAPII, 5. - Imporlance de l'égonlage mantel

A -. Quantités absolues en litres

B - En p. 100 du volume de lait obtenu a la machine (traite et égouttage machine)

Nous étudierons successivement l'influence des différents facteurs zootechniques sur les quantités absolues.

Io) Numéro d'ordre de la lactation. Répétabilité.

Alors que les productions laitières enregistrées pour les brebis avec I et 2 agneaux passent en moyenne de 87 en $\mathrm{I}^{\mathrm{re}}$, à 92 en $2^{\mathrm{e}}$ et $\mathrm{IO} 2$ litres en $3^{\mathrm{e}}, 4^{\mathrm{e}}$ et $5^{\mathrm{e}}$ lactation, le 


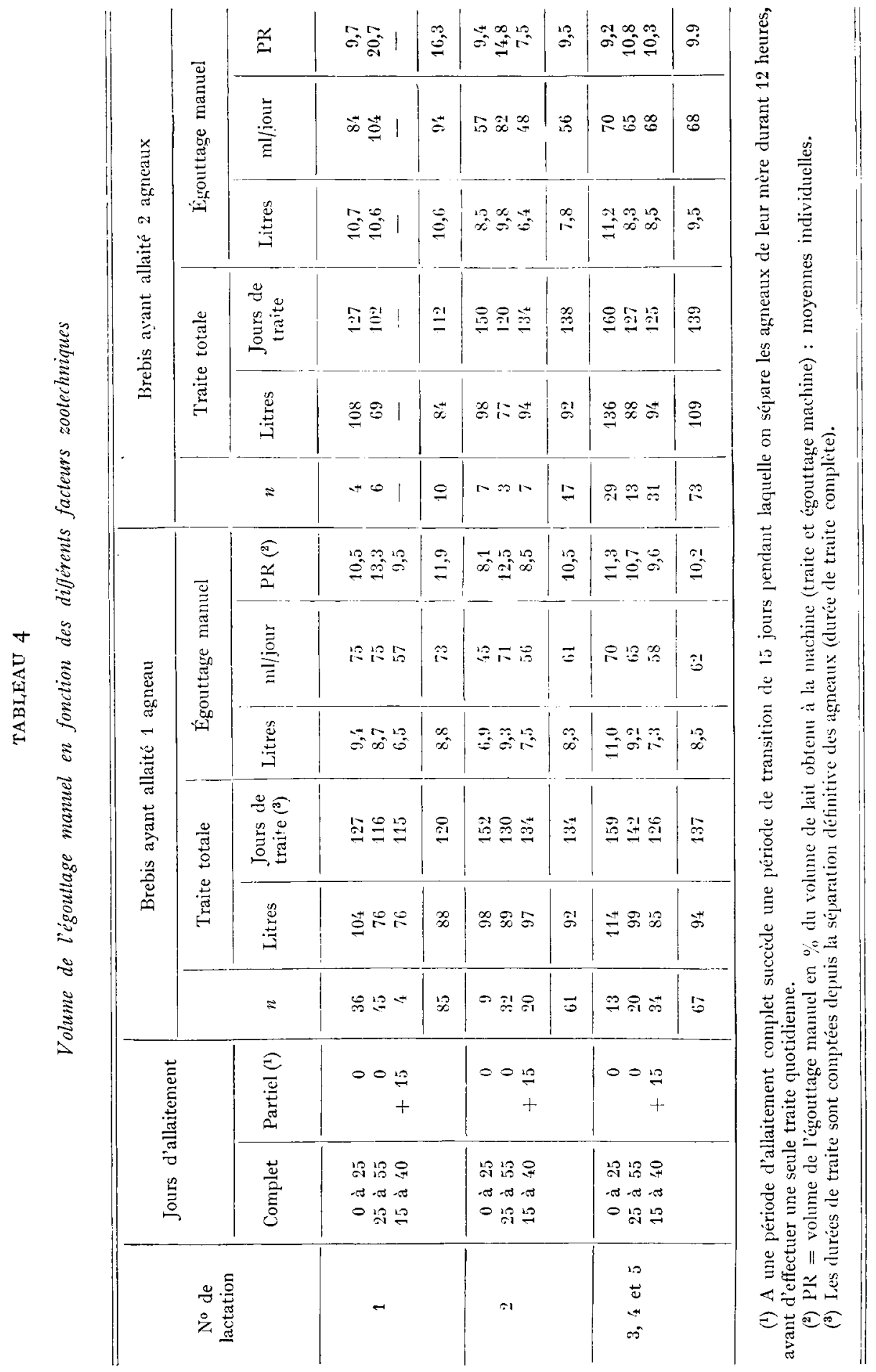


volume total de la repasse reste voisin de 9 litres (tableau 4). En calculant le volume moyen journalier, pour éliminer les différences dans la durée des périodes de traite (nous verrons plus loin que la quantité de lait obtenue à l'égouttage manuel est pratiquement constante au cours de la lactation), on constate cependant un volume

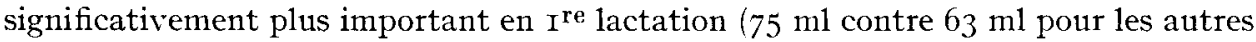
lactations: $\mathrm{P}<0,05)$.

Pour calculer la répétabilité de ce volume, nous avons utilisé les données de 98 brebis contrôlées pendant plus de 2 lactations. Les calculs ont été effectués sur les données brutes, sur les données corrigées pour le $n^{\circ} \mathrm{d}^{\prime}$ ordre de la lactation (- I $2 \mathrm{ml}$ de lait par jour pour les premières lactations) et sur les données transformées en log après application de la correction précédente.

Les coefficients de répétabilité qui résultent de l'analyse de variance du tableau 5 , sont de 0,28 sans correction des données, de 0,53 après correction et de $0,5 \mathrm{I}$ après correction et transformation $\log$. I e volume moyen de l'égouttage manuel est donc un caractère assez peu répétable d'une lactation à l'autre, surtout sil'on tient compte du fait que les erreurs de mesure ont été réduites au minimum, puisqu'il s'agit de productions enregistrées quotidiennement.

\section{TABLEAT 5}

Répélabilité du zolune de l'égouttagé manuel

\begin{tabular}{|c|c|c|c|c|c|}
\hline \multicolumn{6}{|c|}{ 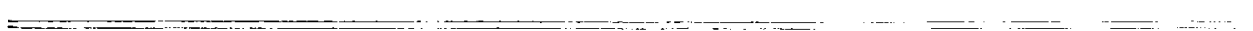 } \\
\hline \multirow[b]{2}{*}{ Source de variation } & \multirow[b]{2}{*}{ d l } & \multicolumn{3}{|c|}{ Carré moyen } & \multirow[b]{2}{*}{$\begin{array}{l}\text { Lstimation de } \\
\text { la variance }\end{array}$} \\
\hline & & $\begin{array}{c}\text { Sans } \\
\text { correction }\end{array}$ & $\begin{array}{c}\text { Apris } \\
\text { correction }\end{array}$ & $\begin{array}{l}\text { Correction } \\
\text { et } \operatorname{logr}_{x}\end{array}$ & \\
\hline Totale & 242 & 710 & 692 & $7,51 \mathrm{i}$ & \\
\hline Entre brebis & 97 & 1005 & $1: 45$ & 5,316 & $\sigma_{i}^{2}+2,47 \sigma_{\varphi}^{2}$ \\
\hline Intra brebis & 145 & 512 & 322 & $2,2 \geq 29$ & \\
\hline \multicolumn{2}{|c|}{ Coefficient de répétabilité } & $0,2 x$ & 0,53 & 0,51 & \\
\hline
\end{tabular}

$2^{\circ}$ ) Date de mise à la traite.

Nous n'avons pas observé d'influence de la durée d'allaitement sur la quantité moyenne journalière de lait recueilli à l'égouttage manuel (tableau 4).

\section{$\left.3^{\circ}\right)$ Mode de sevrage des agneaux.}

Le fait d'habituer les brebis à la traite machine pendant les 2 semaines du sevrage progressif ne permet pas de réduire l'importance de l'égouttage manuel (tableau 6). 
TABIEAU 6

Infuence du mode de sevrage (brutal ou progressif) sur le volume de l'égouttage manuel brebis avec $\mathrm{I}$ agneau

\begin{tabular}{|c|c|c|c|c|c|}
\hline \multirow{2}{*}{ Année } & \multirow{2}{*}{ Effectif } & \multicolumn{2}{|c|}{ Durée d'allaitement (jours) } & \multirow{2}{*}{$\begin{array}{l}\text { Durée de traite } \\
\text { en jours }\end{array}$} & \multirow{2}{*}{$\begin{array}{l}\text { Egouttage } \\
\text { manuel (litres) }\end{array}$} \\
\hline & & Total & l'articl & & \\
\hline 1958 & $\begin{array}{r}10 \\
7\end{array}$ & $\begin{array}{l}42 \\
12\end{array}$ & 15 & $\begin{array}{l}140 \\
140\end{array}$ & $\begin{array}{l}9,4 \\
9,8\end{array}$ \\
\hline 1959 & $\begin{array}{l}15 \\
13\end{array}$ & $\begin{array}{l}14 \\
21\end{array}$ & $11^{\prime \prime}$ & $\begin{array}{l}137 \\
141\end{array}$ & $\begin{array}{l}7,5 \\
7,2\end{array}$ \\
\hline
\end{tabular}

$\left.4^{\circ}\right)$ Nombre d'agneaux allaités.

Les données globales du tableau 4 , ne font pas apparaître de différences entre brebis ayant allaité $\mathrm{I}$ et 2 agneaux sauf en Ire lactation. En effectuant les comparaisons entre lots par ailleurs identiques (tableau 7 ), nous constatons que les bessonnières cèdent moins bien leur lait à la traite que les brebis ayant donné des simples. Cette différence de I à 2 litres n'est cependant pas significative.

\section{TAMLEAU 7}

Influence du nombre d'agneaux allaités sur le volume de l'égouttage manuel

\begin{tabular}{|c|c|c|c|c|c|c|}
\hline Année & Effectif & $\begin{array}{l}\text { Agneaux } \\
\text { allaités }\end{array}$ & $\begin{array}{c}\text { Durée d'allai- } \\
\text { tement }(\mathrm{j})\end{array}$ & $\begin{array}{l}\text { Durce de } \\
\text { traite } \\
\text { complite (i) }\end{array}$ & $\begin{array}{l}\text { Egouttage } \\
\text { manuel (1) } \\
\text { (litres) }\end{array}$ & $\begin{array}{c}\text { Différence } \\
\text { en litres }\end{array}$ \\
\hline 1957 & $\begin{array}{r}13 \\
5\end{array}$ & $\begin{array}{l}1 \\
2\end{array}$ & $\begin{array}{l}42 \\
42\end{array}$ & $\begin{array}{l}98 \\
98\end{array}$ & $\begin{array}{rr}7,6 & (\mathrm{x}) \\
9,4 & (20)\end{array}$ & $+1,8$ \\
\hline & $\begin{array}{l}10 \\
21\end{array}$ & $\begin{array}{l}1 \\
2\end{array}$ & 7 & $\begin{array}{l}161 \\
152\end{array}$ & $\begin{array}{ll}8,1 & (20) \\
9,6 & (2 \prime)\end{array}$ & $\div 1,5$ \\
\hline & $\begin{array}{l}15 \\
13\end{array}$ & $\begin{array}{l}1 \\
2\end{array}$ & $\begin{array}{l}28 \\
26\end{array}$ & $\begin{array}{l}137 \\
142\end{array}$ & $\begin{array}{ll}7,5 & (7) \\
8,8 & (8)\end{array}$ & $+1,3$ \\
\hline
\end{tabular}

(1) Tous avons indiqué entre parenthises, la frécuence des observations supérieures à 12 litres.

$\left.5^{\circ}\right)$ Traite du matin et traite du soir.

Lorsque l'intervalle est en moyenne de $\mathrm{I} 2$ heures, nous constatons que le volume de l'égouttage manuel du matin représente 53,6 p. Ioo du volume de la repasse totale journalière. Cependant, compte tenu du fait que la traite du matin fournit $5 \mathrm{I}, 9 \mathrm{p}$. Ioo du volume de la traite quotidienne, le pourcentage de lait obtenu à l'égouttage manuel du matin, n'est plus significativement supérieur à celui du soir (o,8 p. Ioo en plus). 


\section{II. - Évolution de l'égouttage manuel au cours de la lactation}

Io) Quantités absolues.

Pour des brebis soumises à la traite machine dès leur I $^{\text {re }}$ lactation, quelle que soit la durée d'allaitement, on peut distinguer deux phases :

- Une phase de décroissance d'allure hyperbolique portant sur les trois premières semaines de traite : la quantité moyenne journalière de lait à l'égouttage manuel passe de $190 \mathrm{ml}$ au $\mathrm{I}^{\mathrm{er}}$ jour de traite, à $\mathrm{I} 26 \mathrm{ml} \mathrm{au} 4^{\mathrm{e}}$ jour, puis à $80 \mathrm{ml}$ au $2 \mathrm{I}^{\mathrm{e}}$ jour ;

- Un plateau à partir de la $4^{\mathrm{e}}$ semaine $(70 \mathrm{ml})$ suivi d'une diminution lente jusqu'en fin de lactation (50 à $60 \mathrm{ml}$ ).

La comparaison de lots contemporains et identiques sauf en ce qui concerne le facteur étudié, montre que le volume de l'égouttage manuel n'est pas influencé par les facteurs suivants:

durée d'allaitement (graphique 6, courbes I et II).

mode de sevrage (graphique 6 , courbes VI et VII).

Pour les bessonnières, l'égouttage manuel présente une évolution identique à celle des brebis avec simples, mais se situe à un niveau légèrement supérieur (graphique 6 , courbes III, IV, V).

$\left.2^{\circ}\right)$ Pourcentage de lait recueilli à l'égouttage manuel (P. R.).

Ce pourcentage, très élevé au cours des premiers jours de traite ( I 8 p. Ioo pour les brebis en Ire lactation, I 2 p. IOO pour les brebis adultes), décroît pendant les 2 semaines suivante ( 6 à 8 p. Ioo), puis augmente jusqu'en fin de lactation pour atteindre une valeur de $I_{5}$ à 30 p. Ioo suivant les lots. En conclusion, le P. R. n'a de signification que si l'on précise le stade de lactation auquel il a été obtenu.

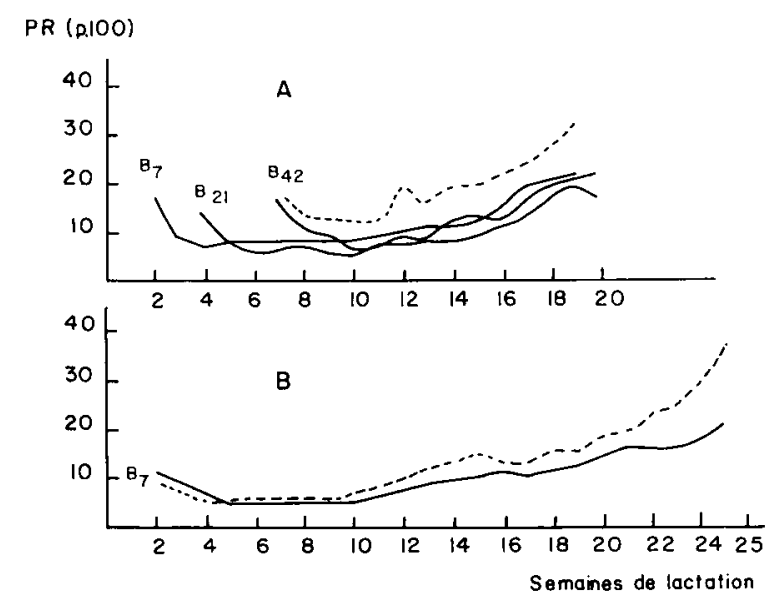

Grapr. 7. - Importance relative du lait de l'goultage machine $(P$. R.) en fonction du stade de lactation A - brebis en $\mathbf{I}^{\text {re lactation }}$

B - - brebis en $3^{\text {e }}$ et $4^{\text {e }}$ lactation trait plein : brebis avec 1 agneau pointillé : brebis avec 2 agneaux 

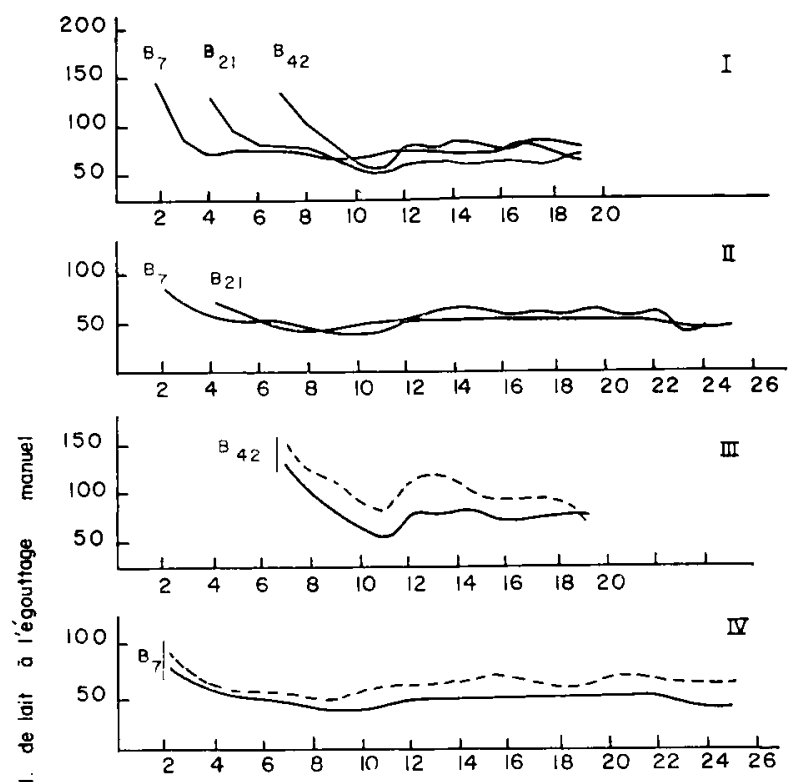

$\overline{\mathbf{E}}$
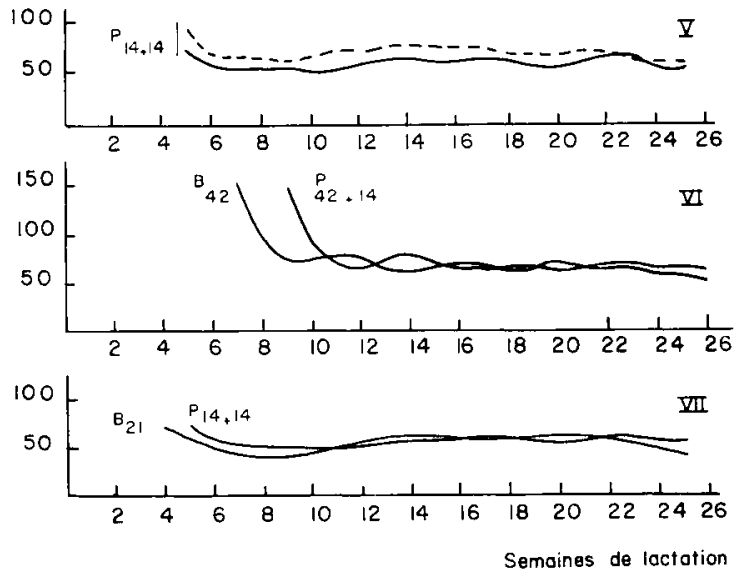

GRAPH. 6. - Variation du volume de l'égoultage manuel au cours de la lactalion, en fonclion de la durée d'allaitement, du nombre d'agneaux allaités et du mode de sevrage $\mathrm{B}_{i}$ - sevrage brutal après $i$ jours d'allaitement

$\mathrm{P}_{i+14}-$ sevrage progressif d'une durée de I4 jours après $i$ jours d'allaitement complet

II - brebis en I $^{\mathrm{re}}$ lactation, avec I agneau durée dallaitement

III — brebis en Ire lactation

IV - brebis en $2^{\mathrm{e}}, 3^{\mathrm{e}}$ et $4^{\mathrm{e}}$ lactation nombre d'agneaux allatiés

VI - brebis en $2^{\mathrm{e}}$ et $3^{\mathrm{e}}$ lactation, avec I agneau
VII - brebis en $2^{\mathrm{e}}, 3^{\mathrm{e}}$ et $4^{\mathrm{e}}$ lactation, avec I agneau mode de sevrage

trait plein : brebis avec I agneau, pointillé : brebis avec: 2 agneaux. 


\section{III. - Variabilité journalière de l'égouttage manuel}

On peut exprimer la variabilité journalière de la quantité de lait obtenue à l'égouttage manuel en calculant la moyenne des différences relatives enregistrées d'un jour à l'autre au cours de la lactation. Les résultats obtenus sur 42 brebis (tableau 8) révèlent des fluctuations journalières considérables. Alors que la variabilité moyenne de la quantité de lait est de l'ordre de 9 p. Ioo, celle de l'égouttage manuel atteint $76 \mathrm{p}$. Ioo.

TABLEAU 8

Coefficient de variabilité journalière du volume de l'égouttage manuel comparé à celui de la traite totale (105 jours de traite)

\begin{tabular}{|c|c|c|c|c|}
\hline & \multicolumn{2}{|c|}{ Traite totale } & \multicolumn{2}{|c|}{ Égouttage manuel } \\
\hline & C.V.J. & $\begin{array}{l}\text { Limites de } \\
\text { variation }\end{array}$ & C.V.J. & $\begin{array}{c}\text { Limites de } \\
\text { variation }\end{array}$ \\
\hline A & 8,5 & 5,3 à 14,7 & 76,0 & $2 x$ à 146 \\
\hline B & 7,6 & $6,2 \dot{\mathrm{a}} \quad 9,1$ & - & - \\
\hline
\end{tabular}

A : 42 brebis traites ì la machine.

B : 16 brebis traites manuellement.

\section{IV. - Richesse en matière grasse du lait obtenu à l'égouttage manuel}

Le taux butyreux du lait de l'égouttage manuel est r,5 à 2 fois plus élevé que celui du lait machine. Nos résultats sont conformes à ceux de SALERNo (I935 : Brebis Gentile de Puglia) et Grunfel, et al. (I958 : Brebis Awasi). Ces auteurs obtiennent respectivement un taux butyreux (en $\mathrm{g}$ p. Ioo g de lait) de 8,5 et 5,9g pour le lait de la première traite - de II,4 et $8,7 \mathrm{~g}$ pour le lait de l'égouttage manuel.

Des résultats portés sur le tableau 9 , nous pouvons tirer les remarques suivantes:

$\mathrm{I}^{\mathrm{o}}$ ) pour les 2 fractions de la traite (lait machine et égouttage manuel) l'évolution de la richesse en matière grasse se traduit par un accroissement rapide de la $8 \mathrm{e}$ à la $20^{\mathrm{e}}$ semaine de lactation ;

$\left.2^{\circ}\right)$ avec le déroulement de la lactation, le lait de l'égouttage manuel s'enrichit moins vite en matière grasse que le lait trait à la machine : le rapport des taux butyreux est de $I, 96$ à la $8^{\mathrm{e}}$ semaine contre $\mathrm{I}, 54$ à la $28^{\mathrm{e}}$ semaine de lactation ;

$\left.3^{\circ}\right)$ 1'écart type des taux butyreux du lait machine augmente avec le déroulement de la lactation, par contre celui de l'égouttage manuel diminue pendant la même période ;

$\left.4^{\circ}\right)$ la quantité totale de matière grasse obtenue à l'égouttage manuel représente 23 à 3 I p. Ioo de celle obtenue à la traite machine.

Ces remarques (jointes aux observations du $\S$ II) montrent l'intérêt que l'on doit attribuer à l'égouttage manuel. Dans le cadre du contrôle laitier, le fait de le 
négliger est susceptible d'entrainer des erreurs par défaut importantes, principalement dans le cas où le premier contrôle se situe lors des premiers jours de traite à la machine. Ces erreurs sont d'ailleurs difficiles à corriger, puisqu'il n'existe aucune relation entre les productions de matière grasse de l'égouttage manuel et de la traite machine.

$$
\text { TABLEAU } 9
$$

Richesse en matière grasse du lait obtenu à la machine et à l'égouttage manuel

\begin{tabular}{|c|c|c|c|c|c|c|c|c|c|c|}
\hline \multirow{2}{*}{ Tours de lactation } & \multirow[b]{2}{*}{$n$} & \multicolumn{3}{|c|}{$\mathrm{TB}_{1}$} & \multicolumn{3}{|c|}{$\mathrm{TB}_{2}$} & \multirow{2}{*}{$\mathrm{r}$} & \multirow{2}{*}{$\frac{\mathrm{TB}_{1}}{\mathrm{~TB}_{2}}$} & \multirow{2}{*}{$\begin{array}{c}\mathrm{PR} \\
\text { matière } \\
\text { grasse }\end{array}$} \\
\hline & & $\ddot{x}$ & $\sigma$ & C.V & $\bar{x}$ & $\sigma$ & $\mathrm{C.V}$ & & & \\
\hline 50 à 56 & 65 & 56,4 & 9,6 & 17,8 & 110,4 & 17,5 & 15,4 & 0,29 & 1,99 & 29,5 \\
\hline 78 à $\quad 8 f_{4}$ & 119 & 60,1 & 9,8 & 16,6 & 115,0 & 15,9 & 13,9 & 0,45 & 1,91 & 23,7 \\
\hline 106 à 112 & 113 & 73,5 & 12,1 & 16,2 & 131,5 & 14,0 & 10,6 & 0,49 & 1,78 & 31,4 \\
\hline 134 à 140 & 108 & 91,0 & 16,7 & 18,6 & 148,1 & 15,5 & 10,8 & 0,56 & 1,63 & 28,9 \\
\hline 161 à 168 & 72 & 97,1 & 16,2 & 16,4 & 152,7 & 13,9 & 9,1 & 0,59 & 1,57 & 25,9 \\
\hline \multirow[t]{2}{*}{189 à 196} & 19 & 96,4 & 16,1 & 16,6 & $1 / 48,1$ & 10,7 & 7,4 & 0,55 & 1,54 & 29,9 \\
\hline & & & & & & & & Moy : 0,52 & & \\
\hline
\end{tabular}

$\mathrm{r}$ : coefficient de corrélation entre $\mathrm{TB}_{1}$ et $\mathrm{TB}_{2}$

$\mathrm{TB}_{1}$ : taux butyreux du lait obtenu à la machine (lait de la traite machine proprement dite et de l'égouttage machine) en g p. $1000 \mathrm{~g}$. de lait.

$\mathrm{TB}_{2}$ : taux butyreux du lait obtenu à l'égouttage manuel en g p. $1000 \mathrm{~g}$. de lait.

moyenne $(\bar{x})$; écart-type $(\sigma)$; coefficient de variation (C.V).

PR matière grasse : matière grasse du lait de l'égouttage manuel en p. 100 de celle du lait obtenu à la machine.

\section{V. - Action de l'égouttage manuel sur le maintien de la sécrétion lactée}

Afin d'estimer le rôle de l'égouttage manuel, nous avons comparé la production laitière de brebis traites avec égouttage (vidange normale) à celle de brebis traites sans égouttage (hypo-vidange) ou avec 2 égouttages consécutifs (hyper-vidange).

$\left.\mathrm{I}^{\circ}\right)$ Hypo-vidange : traite à la machine sans égouttage manuel.

90 brebis adultes ont été réparties en 3 lots différents par leur date de sevrage : I er lot: traite depuis l'agnelage ;

$2^{\mathrm{e}}$ lot $: 2$ semaines d'allaitement complet suivies de 2 semaines de sevrage progressif ;

$3^{\mathrm{e}}$ lot : sevrage brutal après 3 semaines d'allaitement complet.

Au moment de la mise à la traite définitive, chaque lot est divisé en 2 : une moitié est traite selon la méthode classique, pour l'autre moitié l'égouttage manuel est supprimé $\left({ }^{1}\right)$.

(1) Pour les brebis privées d'égouttage manuel et uniquement pour celles-ci, on procédait avant la traite au massage de la mamelle avec un linge humide et tiède. 
Pour les trois groupes, l'absence d'égouttage manuel entraîne une réduction de 2 à 3 semaines de la durée de lactation. Il est cependant plus intéressant de constater que la production laitière totale a été réduite de 26,4 litres ( 29 p. Ioo de la production du lot témoin) alors que l'égouttage manuel des lots témoins ne représente que 7 à 8 litres. En ramenant la durée de traite des lots " avec égouttage " au niveau de celle des lots " sans égouttage ", la différence totale n'est plus que de 20, I litres (23 p. Ioo de la production du lot témoin). Cette différence correspond à un écart journalier de $I 55 \mathrm{ml}$, à peu près constant quel que soit le stade de lactation. Il en résulte que le coefficient de persistance de la lactation des brebis traites sans égouttage manuel est inférieur à celui des autres brebis.

TABI,EAU IO

Traite avec ou sans égoultage mamuel

\begin{tabular}{|c|c|c|c|c|c|c|c|c|c|}
\hline \multicolumn{2}{|c|}{$\begin{array}{l}\text { Jours } \\
\text { d'allaitement }\end{array}$} & \multirow{2}{*}{$\begin{array}{l}\text { Avec ou } \\
\text { sans } \\
\text { égouttage }\end{array}$} & \multirow{2}{*}{ liffectif } & \multirow{2}{*}{$\begin{array}{l}\text { Production } \\
\text { totale en } \\
\text { litres }\end{array}$} & \multirow{2}{*}{$\begin{array}{l}\text { Jours de } \\
\text { lactation }\end{array}$} & \multicolumn{2}{|c|}{ Persistance $\left({ }^{1}\right)$} & \multirow{2}{*}{ PR } & \multirow{2}{*}{$\begin{array}{l}\text { Égouttage } \\
\text { manuel } \\
\text { en litres }\end{array}$} \\
\hline Total & Partiel & & & & & $\mathrm{CP}_{2}$ & $\mathrm{CP}_{3}$ & & \\
\hline \multirow[t]{2}{*}{6} & \multirow[t]{2}{*}{0} & \multirow[t]{2}{*}{$\begin{array}{l}\text { avec } \\
\text { sans }\end{array}$} & $\begin{array}{l}10 \\
13\end{array}$ & $\begin{array}{r}109,6 \\
81,5\end{array}$ & $\begin{array}{l}168 \\
151\end{array}$ & $\begin{array}{l}0,9 y_{1}^{\prime} \\
0,917\end{array}$ & $\begin{array}{l}0,936 \\
0,9 \pm 2\end{array}$ & \multirow[t]{2}{*}{8,2} & \multirow[t]{2}{*}{$\frac{8,1}{-}$} \\
\hline & & & différence & $2 x, 1\left({ }^{2}\right)$ & 17 & 0,027 & $0,01^{\prime} t$ & & \\
\hline \multirow[t]{2}{*}{$1^{\prime} t$} & \multirow[t]{2}{*}{$1 ' t$} & \multirow[t]{2}{*}{$\begin{array}{l}\text { avec } \\
\text { sans }\end{array}$} & $\begin{array}{l}15 \\
14\end{array}$ & $\begin{array}{l}85,6 \\
60,6\end{array}$ & $\begin{array}{l}165 \\
147\end{array}$ & $\begin{array}{l}0,936 \\
0,901\end{array}$ & $\begin{array}{l}0,92^{\prime} \\
0,89 \overline{5}\end{array}$ & \multirow[t]{2}{*}{10,1} & \multirow[t]{2}{*}{$\underline{7,5}$} \\
\hline & & & différence & $25,0\left({ }^{2}\right)$ & 18 & 0,035 & 0,029 & & \\
\hline \multirow[t]{2}{*}{21} & \multirow[t]{2}{*}{0} & $\begin{array}{l}\text { avec } \\
\text { sans }\end{array}$ & $\begin{array}{l}13 \\
15\end{array}$ & $\begin{array}{l}83,2 \\
57,2\end{array}$ & $\begin{array}{l}162 \\
1 \div 4\end{array}$ & $\begin{array}{l}0,947 \\
0,898\end{array}$ & $\begin{array}{l}0,9 / 2 \\
0,91 ; 1\end{array}$ & \multirow[t]{2}{*}{10,2} & \multirow[t]{2}{*}{$\begin{array}{l}7,9 \\
-\end{array}$} \\
\hline & & & différence & $26,0\left({ }^{2}\right)$ & 18 & $0,0 \div 9$ & $0,0 \div 28$ & & \\
\hline
\end{tabular}

(1) Coefficients de persistance hebdomadaires des périodes 2a et :3. (cf. tableau 1).

(2) Différence significative au seuil $\mathrm{P}=0,01$.

Nous n'avons relevé aucune différence entre la richesse en matière grasse du lait obtenu à la traite habituelle et celle du lait obtenu à la traite sans égouttage : 34 prélèvements de lait de mélange donnent des taux butyreux respectivement de 6,7 et $6,6 \mathrm{~g}$ p. Ioo $\mathrm{g}$ de lait.

$\left.2^{\circ}\right)$ Hyper-vidange : traite à la machine suivie de 2 égouttages manuels.

Lot expérimental : $\mathrm{I}_{4}$ brebis séparées de leurs agneaux 4 à 6 jours après la mise bas et soumises à chaque traite à 2 égouttage manuels.

Lot témoin : 49 brebis séparées définitivement de leurs agneaux à 30 jours. 
Ces 2 lots, composés d'animaux de même âge moyen $\left(35 \mathrm{p}\right.$. Ioo en $2^{\mathrm{e}}, 4^{2} \mathrm{p}$. Ioo en $3^{\text {e }}$ et 2 I p. Ioo en $4^{\mathrm{e}}$ lactation) ne sont pas comparables en ce qui concerne la durée d'allaitement, mais nous avons montré (RICORDEAU et DENAMUR, I962) que ce facteur n'avait aucune influence sur le niveau de production laitière ultérieur, exception faite des 20 premiers jours de traite.

En tenant compte de la production laitière obtenue entre le $50^{\mathrm{e}}$ et le $\mathrm{I}_{3 \mathrm{O}^{\mathrm{e}}}$ jour de lactation, on enregistre une augmentation significative de 8,7 litres (I4,8 p. IOO de la production de lot témoin) en faveur du lot expérimental. Cette différence correspond à un écart journalier de Ioo $\mathrm{ml}$, à peu près constant quel que soit le stade de lactation. On peut noter également que l'égouttage supplémentaire s'est traduit par un accroissement de production de la traite machine et des égouttages.

TABLEAU I I

Traite suivie d'un et de deux égouttages manuels: $5^{\circ}$ au $13^{\circ}$ jour de lactation

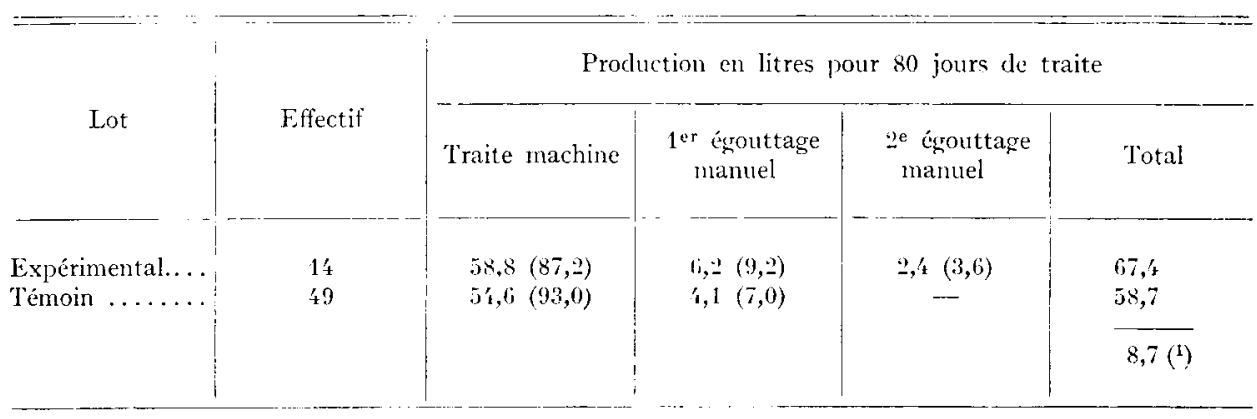

(1) Différence significative. $\mathrm{P}=0,08$.

Entre parenthises, le volume de lait en p. 100 du volume de la traite totale.

\section{D. - LAIT RÍSIDUEL,}

Le lait résiduel est estimé immédiatement après l'égouttage manuel, par la quantité de lait que permet d'obtenir l'injection intraveineuse d'une U. I. d'ocytocine $\left({ }^{1}\right) .32$ brebis ont subi ce traitement I fois par semaine après la traite du soir (tableau 2).

La quantité de lait résiduel est I, 7 fois plus importante que celle de l'égouttage manuel, mais évolue de façon comparable. Ein valeur relative, elle représente I 3 p. roo du total de la traite classique du soir au $2^{\mathrm{e}}$ mois et atteint 20 à $23 \mathrm{p}$. Ioo en fin de période de traite.

En ce qui concerne la richesse en matière grasse, 2 contrôles effectués au cours $\mathrm{du} 3^{\mathrm{e}}$ mois de lactation donnent les résultats suivants en $\mathrm{g} \mathrm{p}$. Iooo $\mathrm{g}$ de lait:

$5,8 \mathrm{~g}$ pour le lait de la traite machine (matin et soir) ;

I2, I g pour le lait de l'égouttage manuel (matin et soir) ;

I4,6 g pour le lait résiduel (soir).

(1) Précisons que cette dose de I L. I. d'ocytocine est arbitraire, puisque nous avons montré par ailleurs (DENANLR et MARTINET, Ig62) qu'il existait une relation linéaire entre le "lait résiduel "exprimé en p. 100 du volume total de lait extrait et le log de la dose d'ocytocine employée (o, i d̀ 5 C. I.). 
SEMJAN (I962) obtient des résultats comparables, mais en pratiquant des injections sous-cutanées de 6 à Io U. I. d'extrait post-hypophysaire, 5 à 7 minutes après la traite ordinaire: sur 27 brebis contrôlées du $4^{\mathrm{e}}$ au $8^{\mathrm{e}}$ mois de lactation, le lait résiduel représente $76 \mathrm{ml}$ (à $\mathrm{I} 2,4 \mathrm{~g}$ de matière grasse) pour $227 \mathrm{ml}$ de lait (à $7,8 \mathrm{~g}$ de matière grasse) à la traite ordinaire.

\section{E. - CORRÉlations ENTRE LES PRINCIPAUX PARAMÈtTES DE TRAITE ET DE LACTATION}

De 1'ensemble des résultats obtenus sur les 32 brebis contrôlées régulièrement $\mathrm{du} 64^{\mathrm{e}}$ à $\mathrm{r} 64^{\mathrm{e}}$ jour de lactation, nous avons calculé les relations suivantes :

TABLEAU I2

Corrélations entre les principales caractéristiques de traite ( 32 brebis)

\begin{tabular}{|c|c|c|c|c|c|c|c|c|c|}
\hline 2 & 3 & 4 & 5 & 6 & 7 & 8 & 9 & 10 & Variables \\
\hline 0,$6 ; * *$ & 0,10 & $\begin{array}{l}0,19^{* *} \\
0, \geq 0\end{array}$ & $\begin{array}{r}0,21 \\
-0,01\end{array}$ & $\begin{array}{l}0,21 \\
0,16 \\
0,49^{* *}\end{array}$ & $\begin{array}{l}0,06 \\
0,21 \\
0,10 \\
0,55^{* *}\end{array}$ & $\begin{array}{l}0,38^{* *} \\
0,50^{* *} \\
0,08 \\
0,07 \\
0,15\end{array}$ & $\begin{array}{l}0,39 * * \\
0,10 \\
0,22 \\
0,20 \\
0,03\end{array}$ & $\begin{array}{r}0,25 \\
0,23 \\
0,16 \\
-0,01 \\
0,04\end{array}$ & $\begin{array}{c}1: \text { Volume total de lait en } \\
100 \text { jours } \\
2: \text { Volume des } 15 \text { premières } \\
\text { secondes de traite } \\
5: \text { Volume de l'égouttage ma- } \\
\text { chine } \\
\text { 6: Volume de l'égouttage manuel } \\
\text { i: Volume de lait résiduel }\end{array}$ \\
\hline
\end{tabular}

Variables $3:$ p. 100 de lait obtenu au cours de la première minute de traite machine.

i : durée de la traite machine proprement dite.

$x$ : rurée de lactation.

9 : persistance $\mathrm{P}_{2-3}$ (cf. tableau 1).

10 : persistance $\mathrm{CP}_{2+3}$ (cf. tableau 1 ).

** : significutif au sauil $\mathrm{P}=0,01$.

$\left.I^{\circ}\right)$ La quantité de lait obtenue au cours des I $_{5}$ premières secondes de traite est en corrélation positive avec le niveau de production et la durée de lactation, mais elle est indépendante du volume de l'égouttage machine et de l'égouttage manuel. Cette quantité peut être retenue comme critère de sélection.

$2^{\circ}$ ) La durée de la traite à la machine est en corrélation positive avec la quantité de lait par lactation.

$3^{\circ}$ ) Il n'existe aucune relation significative entre le niveau de production laitière et le pourcentage de lait récolté durant la première minute de traite, ce qui tend à montrer que les brebis les plus rapides à traire ne sont pas toujours les meilleures laitières.

$4^{\circ}$ ) Il n'existe aucune corrélation significative entre la production laitière totale d'une part, le volume de lait obtenu à l'égouttage machine, à l'égouttage manueI et le volume de lait résiduel d'autre part. 
$\left.5^{\circ}\right)$ La quantité de lait obtenue à l'égouttage manuel est liée au volume de l'égouttage machine et au volume de lait résiduel.

$6^{\circ}$ Le coefficient de persistance hebdomadaire calculé du $5^{\circ}$ au ${ }^{\circ} 5^{\mathrm{e}}$ jour de lactation $\left(\mathrm{CP}_{\mathbf{2}^{+3}}\right)$ est pratiquement indépendant du volume de l'égouttage machine, de l'égouttage manuel et de lait résiduel.

\section{DISCUUSSION}

Les principes qui inspirent les essais entrepris pour améliorer la traite des brebis peuvent se résumer ainsi :

Traire le maximum de lait à la machine dans le minimum de temps ;

Simplifier, ou mieux, supprimer l'égouttage manuel qui représente la sujétion la plus importante et la plus coûteuse.

Il va de soi que ces améliorations ne doivent pas entrainer de diminution de la sécrétion lactée, mais au contraire favoriser l'accroissement de la production moyenne du cheptel laitier.

\section{Traire le maximum de lait à la machine dans le minimum de temps.}

L'étude préliminaire de la cinétique de descente du lait au cours de la traite machine qui figure au début de cet article nous permet de distinguer (après le $60^{\mathrm{e}}$ jour de lactation) deux phases:

Une phase d'écoulement du lait citernal qui dure I5 à 30 secondes ;

Une phase d'écoulement à débit plus rapide, située entre la $30^{\mathrm{e}}$ et la $45^{\mathrm{e}}$ seconde de traite et qui correspondrait à l'arrivée du lait alvéolaire chassé par le réflexe neuro-endocrinien d'éjection.

Les origines attribuées à 1'une et l'autre phase paraissent logiques; elles sont vraisemblablement trop schématiques et devront être précisées avec plus de rigueur en utilisant notamment une technique d'enregistrement plus précise.

Nous avons pu en outre constater au cours de la traite machine : que la quantité de lait obtenue au cours des premières fractions de traite est en relation étroite avec le niveau de production de la brebis, qu'il est possible de réduire la durée de traite machine, puisque les quantités de lait obtenues au-delà d'une minute sont faibles ( $8 \mathrm{p}$. Ioo du volume de traite machine).

Nous avons enregistré la cinétique de la descente du lait, mais cette cinétique est, pour des conditions technologiques données, la résultante de facteurs physiologiques (réllexe neuro-endocrinien d'éjection principalement) et morphologiques. I1 s'en suit que l'interprétation des courbes de descente du lait sera toujours très délicate si l'on ne parvient pas à dissocier et à mesurer les influences morphologiques et physiologiques.

Outre la mise au point d'appareils et de techniques qui permettraient d'estimer le diamètre, l'élasticité du trayon dans différentes conditions de traite, il est essentiel de trouver une mesure pratique, utilisable sur l'animal normal, et capable par exemple 
de déterminer l'amplitude de variation de la pression intra-mammaire attribuable au réflexe neuro-endocrinien d'éjection. La résolution de ce problème permettra de préciser (ce que nous n'avons pu faire dans cette étude), l'importance du réflexe chez un animal donné, son évolution au cours de la lactation, sa variabilité quotidienne et d'envisager une sélection efficace sur ce caractère prépondérant de l'aptitude à la traite.

\section{Simplifier, ou mieux, supprimer l'égouttage manuel.}

En ce qui concerne l'égouttage manuel, les perspectives sont certainement moins encourageantes. Nous savons que la corrélation phénotypique entre le volume de l'égouttage manuel et le volume total de la traite est pratiquement nulle, que la quantité de lait obtenue à l'égouttage machine est un critère assez peu répétable, moins répétables en particulier que la quantité de lait obtenue à la traite machine (RICORDEAU, I963).

La suppression de l'égouttage manuel se traduit, comme nous l'avons vu, par une diminution importante de la production laitière. Par contre, on peut simplifier cet égouttage en supprimant le soubattage $\left({ }^{1}\right)$, ce qui économise $25 \mathrm{p}$. Ioo du temps moyen nécessaire à cette opération, comme l'ont montré les travaux de Bosc (I962) sur brebis Lacaune.

Dans l'état de méconnaissance profonde où nous nous trouvons de la physiologie de la traite des brebis et de l'emploi rationnel des machines à traire, on ne peut envisager sur le plan pratique (tant que ces lacunes ne seront pas comblées) que 2 méthodes pour essayer d'obtenir une amélioration génétique de l'aptitude des brebis à la traite.

Sélection des animaux sur leur aptitude à céder la plus grande part de leur lait à la machine ;

Sélection des animaux sur la quantité d'égouttage manuel.

Dans le premier cas, on peut espérer augmenter le débit moyen de la traite, mais non supprimer 1'égouttage manuel. Dans le second, les gains génétiques attendus risquent d'être faibles ou même nuls, s'il se confirme que les quantités de lait obtenues à chaque égouttage manuel sont peu héritables et que la corrélation génétique existant entre les volumes de lait obtenus à la machine et à l'égouttage manuel est nulle ou légèrement positive. De toute façon, il est essentiel, pour apprécier les chances de réussite d'une telle sélection, de connaître d'une manière plus précise les conséquences de la suppression de l'égouttage manuel. La chute de production est-elle moins importante pour les brebis ayant peu d'égouttage manuel? Existe-t-il un certain pourcentage d'animaux, même réduit, sur lesquels il est possible de supprimer l'égouttage manuel sans modifier la production laitière?

Quels que soient les résultats de ces études, il est vraisemblable que les différences existant entre les potentialités réelles des brebis (mises en évidence par la production pendant l'allaitement) et les productions enregistrées à la traite seront encore très importantes. Dès maintenant, on doit donc envisager de les atténuer, soit par le croisement avec des races plus faciles à traire (si elles existent), soit en

(1) Fortes claques données par le trayeur sur la mamelle avec le dos de la main. 
accroissant nos connaissances des mécanismes physiologiques de la traite. C'est dans cette optique que l'on trouve la justification d'un vaste programme de recherches génétiques et physiologiques permettant :

$I^{\circ}$ De définir et mesurer d'une manière précise les critères morphologiques qui déterminent l'aptitude à la traite ;

$2^{o}$ De trouver les stimuli et les conditions d'utilisation de la machine à traire capables d'agir efficacement sur ces critères, afin de rendre inutile la pratique de 1'égouttage manuel et de diminuer l'écart entre la production laitière obtenue à la traite et celle atteinte pendant 1'allaitement;

$3^{\circ}$ De comparer l'aptitude à la traite des brebis de différentes races pures et des produits de croisement.

Reģu pour publication en auril $\mathrm{Ig}_{3}$.

\section{SUMMARY}

MACHINE MIIKING OF PRÉAIL'FS DU SUD EWES. IMPORTANCE OF THE DIFFERENT MILKING OPERATIONS

The Prialpes du Sud ewes used in these expriments were milked by machine twice a day. Each milking consisted of three operations :

I. Machine milking, is in fact, the period between putting on the teat cups and the end of milk let down ;

2. Machine stripping before the removal of the teat-cups;

3. Hand stripping (called the "repasse ").

The importance of the quantity of milk obtained in the course of these three operations, expressed as a percentage of the total amount milked out, varied between the 64th and I 64th days of the lactation, from 68 to $59 \mathrm{p}$. 100 for the machine milking, from 23 to $28 \mathrm{p}$. Ioo for the machine stripping and from 9 to 13 p. Ioo for the hand stripping. Fven after these successive operations the udder is not completely emptied : the volume of residual milk that can be obtained after injecting oxytocin still reprensents $\mathrm{I}_{3}$ to $23 \mathrm{p}$. 100 of the quantity obtained at the previous milking ( 32 ewes on the experiment).

The average length of machine milking is I $m n 26 \mathrm{~s}$ and that of the machine stripping is $32 \mathrm{~s}$.

The weekly recording of the rate of let down of milk from $32 \mathrm{ewes}$, indicated that two phases could be differentiated : the let down, during the first 15 seconds of the milk of the udder cistern and an evacuation phase, resulting from the action of the oxytocin released by the neuro-endocrine reflex of milk injection (from the 3 oth to $45^{\text {th }} \mathrm{s}$ ). 'The importance of the ejection phase diminishes with the lactation stage.

A study of hand stripping was made with the data from 313 lactations. 'The volume of milk obtained by hand stripping over the whole period of milking is, on average 9.3 liters, this represents I I.2 p. too of the amount of milk obtained during the first two milking operations. This level is higher in the first year of lactation, but varies little in the succeeding lactations. The level is not significantly related to the length of suckling period, the method of weaning or the number of lambs suckled.; the daily variability coefficient is $76 \mathrm{p}$. 100 ; the coefficient of repeatability is 0.28 for non corrected data and 0.53 for corrected data.

The quantity of milk obtained each day by hand stripping rapidly decreases during the first two weeks of milking, but remains practically constant subsequently. The butterfat level of hand stripped milk represents 24 to $30 \mathrm{p}$. 100 (varying as a function of the stage lactation) of the fat level of the milk obtained by the machine.

The discontinuance of hand stripping causes an average decrease of $29 \mathrm{p}$. Ioo in the potential yield of ewes that suckled their lambs for i to 4 weeks. On the contrary, the practice of a supplementary hand stripping after every machine milking permits to obtain a yield gain of $5 \mathrm{p}$. 100 .

The relationships between the total production of milk obtained by milking and the principal milking characteristics were studied. 


\section{RÉFÉRENCES BIBLIOGRAPHIQUES}

Bosc J., ig62. Adaptation du système "Arête de Poisson "à la traite mécanique des brebis. Cah. Ing., agron., 164, 29-36.

Denamur R., Martinet J, 195r. Action de l'ocytocine sur la sécrétion du lait de brebis. Ann. Endoci• 22, $776-781$.

Grunfeld J., Volcani R., lixal E., I959. Quelques observations sur l'adaptation de la brebis Awasi à la traite mécanique (en hébreu). Hanoked., 42.

Ricordeau G., Denamur R., i 962. Production laitière des brebis Préalpes du Sud pendant les phases d'allaitement, de sevrage et de traite. Ann. Zootech., 11, 5-38.

Ricordeau G., 1963. Résultats non publiés.

Salerno A., I935. Problemi inerenti all'attuazione del controllo del latte nel grande gregrge ovino. Ann. Isl. sper. zootec. Roma., 13, $2+1-264$.

SEMJAN S., I962. Residual milk of shcep. XVI e congr. inl. I.aiterie. Copenhague A (1), 1 7-22. 